\title{
Molecular Weight Analysis of Water Soluble Poly(phenylene ethynylene)s Using MALDI-TOF MS
}

\author{
Jiatao Wu, ${ }^{1,2}$ Chunyan Tan, ${ }^{1,2}$ Xinwen Zhou, ${ }^{3}$ Ying Tan, ${ }^{1,2}$ Pengyuan Yang, ${ }^{3}$ and Yuyang Jiang ${ }^{2,4}$ \\ ${ }^{1}$ Department of Chemistry, Tsinghua University, Beijing 100084, P. R. China \\ ${ }^{2}$ The M inistry-Province Jointly Constructed Base for State Key Lab-Shenzhen Key Laboratory of Chemical \\ Biology, the Graduate School at Shenzhen, Tsinghua University, Shenzhen 518055, P. R. China \\ ${ }^{3}$ Institutes of Biomedical Sciences, Fudan University, Shanghai 200032, P. R. China \\ ${ }^{4}$ The Department of Pharmacology and Pharmaceutical Sciences, School of M edicine, Tsinghua \\ University, Beijing 100084, P. R. China \\ Correspondence to: C. Tan (E-mail: tancy@sz.tsinghua.edu.cn) \\ ((Additional Supporting Information may be found in the online version of this article.))
}

\begin{abstract}
:
Molecular weights of seven poly(phenylene ethynylene) (PPE) based water-soluble conjugated polyelectrolytes (CPES) obtained through Sonogashira coupling are determined by matrix-assisted laser desorption/ionization time-of-flight mass spectrometry (MALDI-TOF MS). A standard sample preparation protocol is developed to characterise the seven CPEs using 2,5-dihydroxybenzoic acid (DHB) as the matrix (M) and AgTFA as the cationisation reagent (CR). High quality M ALDI mass spectra are obtained at volume mixing ratios (CPE/M/CR) of 5/5/1 for anionic polymers (P1-P4) and 5/50/1 for cationic polymers (P5-P7). Molecular weight, molecular weight distribution, and end-group information are analyzed. The effects of molecular weight of CPEs on optical and quenching properties are also studied.
\end{abstract}

KEYWORDS: CPES; M ALDI-TOF MS; molecular weight determination; optical properties

INTRODUCTION Conjugated polyelectrolytes (CPEs) have attracted much attention due to their excellent optical, electrochemical, and electrical properties, which have enabled them to be widely used in chemo- and bio-sensing. ${ }^{1-4}$ These unique properties of CPEs depend on not only the polymers' structures, ${ }^{5}$ but also polymers' chain lengths, ${ }^{6}$ which can be characterized by molecular weight (MW) and molecular weight distribution (MWD). Some analytical methods, such as gel permeation chromatography $(\mathrm{GPC}){ }^{7-9}$ nuclear magnetic resonance (NMR), ${ }^{10}{ }^{11}$ electrospray ionization mass spectrometry (ESI-MS) $)^{12}$ and dynamic and static light scatterings (DLS/SLS) ${ }^{13}$, have been employed to provide polymers' MW information. ENREF 7 ENREF 7However, these methods showed different limitations in analysing $M W$ of
CPEs. For GPC, it is necessary to find a suitable standard polymer, otherwise the calibration is unreliable. ${ }^{14}$ NM R methods are currently limited to the low-molecular-weight and organic solvent-dissolved polymers. ${ }^{15}$ The prime challenge for DLS and SLS is that the data interpretation is complicated for the lack of knowledge of the morphological features of CPEs. ${ }^{13}$ ESI-MS has an incompatibility that polymers composed of large and entangled macromolecules are not readily converted to gas-phase species, which MS require for a successful analysis. ${ }^{15}$.

As a powerful toolset for the structural investigation of synthetic polymers,_ENREF 15 matrix-assisted laser desorption/ionization

This is the author manuscript accepted for publication and has undergone full peer review but has not been through Mthials: between this version and the Version record. Please cite this article as doi:10.1002/ pola.28650. 
MS) has

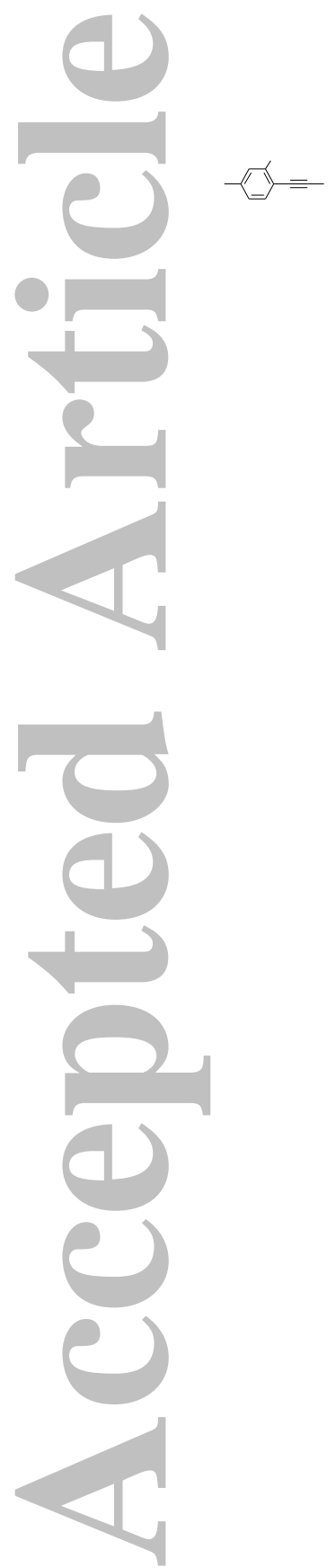


All samples were measured in negative linear ion mode. For all MALDI tests, polymer (P) solutions were prepared at a concentration of $5.0 \mathrm{mg} / \mathrm{mL}$; matrix (M) solutions were prepared at a concentration of $10 \mathrm{mg} / \mathrm{mL}$; and cationization reagent (CR) solutions were prepared at a concentration of $0.1 \mathrm{~mol} / \mathrm{L}$.

TABLE 1 Volume M ixing Ratios for Three Factors: Polymer, Matrix and Cationization Reagent.

\begin{tabular}{|c|c|c|c|}
\hline Run & $V_{P_{1}^{a}}^{a}$ & $V_{M}^{b}$ & $\mathrm{~V}_{C R}^{\mathrm{c}}$ \\
\hline 1 & 1 & 1 & 0 \\
\hline 2 & 1 & 1 & 1 \\
\hline 3 & 1 & 5 & 1 \\
\hline 4 & 5 & 5 & 1 \\
\hline 5 & 5 & 10 & 1 \\
\hline 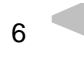 & 5 & 5 & 10 \\
\hline
\end{tabular}

According to Table 1, the solutions were mixed in the given volume mixing ratio, and $1.0 \mu \mathrm{L}$ solution was hand spotted on a stainless steel target and allowed to dry by air. The spectra were analyzed with the operator manually searching for the sample "sweet spot" for data collection. All spectral data were taken from signal averaging of 300 laser shots and processed using Data Explorer 4.5.

\section{RESULTS AND DISCUSSION}

\section{Analysis of MW and MWD}

Since the polymer characterization by Tanaka et al. in $1988{ }^{26} \mathrm{MALDI}$ has become a powerful analytical tool for the characterization of industrial polymer materials. ${ }^{21,27-30}$ Table 2

TABLE 2 MW Information of Seven CPES Obtained by M ALDI-TOF MS

\begin{tabular}{|c|c|c|c|c|}
\hline Polymer & $\overline{M_{n}}(\mathrm{Da})$ & $\overline{M_{w}}(\mathrm{Da})$ & PDI & $\overline{X_{n}}$ \\
\hline P1 & 15319 & 24202 & 1.58 & 62 \\
\hline P2 & 9812 & 14621 & 1.49 & 28 \\
\hline
\end{tabular}

\begin{tabular}{|l|l|l|l|l|}
\hline P3 & 14788 & 22182 & 1.50 & 26 \\
\hline P4 & 14183 & 20968 & 1.48 & 20 \\
\hline P5 & 19796 & 30317 & 1.53 & 29 \\
\hline P6 & 16722 & 26086 & 1.56 & 35 \\
\hline P7 & 15102 & 24918 & 1.65 & 26 \\
\hline & ${ }^{a}$ PDI was obtained according to formula PDI $=\overline{\mathrm{M}_{\mathrm{w}}} / \overline{\mathrm{M}_{\mathrm{n}}}$ \\
\hline
\end{tabular}

shows the characterization data of seven CPEs obtained by MALDI-TOF MS. Generally, the number-average molecular weights $\left(\overline{M_{n}}\right)$ of seven CPEs fall between 9.81 and $19.79 \mathrm{kDa}$, while the weight-average molecular weights $\left(\overline{M_{w}}\right)$ fall within a higher range, between 14.62 and $30.37 \mathrm{kDa}$, resulting in polydispersity indices (PDI) between 1.49 and 1.65, which is reasonable for step-grow th polycondensation. ${ }^{31}$

\section{GPC and MALDI-TOF MS}

The MALDI results of P2 are compared with GPC results, which are shown in Figure 1. Molecular mass determination of $\mathrm{P} 2$ by MALDI-TOF MS and GPC are markedly different on the $\overline{M_{n}}$, $\overline{M_{w}}$, and PDI. The $\overline{M_{w}}$ of P2 determined by GPC ranges from 10,000 to 75,000 Da using polystyrene as standards. The average molecular mass values for $\mathrm{P} 2$ are $\overline{\mathrm{M}_{\mathrm{n}}}=7,103$ $\mathrm{Da}, \overline{\mathrm{M}_{\mathrm{w}}}=22,517 \mathrm{Da}$ and $\mathrm{PDI}=3.17$. Compared with GPC results, MALDI results are skewed toward lower MW. The discernible oligomer distribution ranges from approximately 4,531 (n $=13)$ to above $17,380 \mathrm{Da}(\mathrm{n}>50)$, with the maxima peak intensity at $\mathrm{m} / \mathrm{z} 7440.44$, corresponding to the $20 \mathrm{RUS}$. The mean polymer masses obtained from MALDI MS for P2 are $\overline{M_{n}}=9747 \mathrm{Da}$ and $\overline{M_{w}}=14621 \mathrm{Da}$, with $\mathrm{PDI}=$ 1.49 .

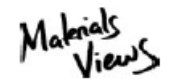



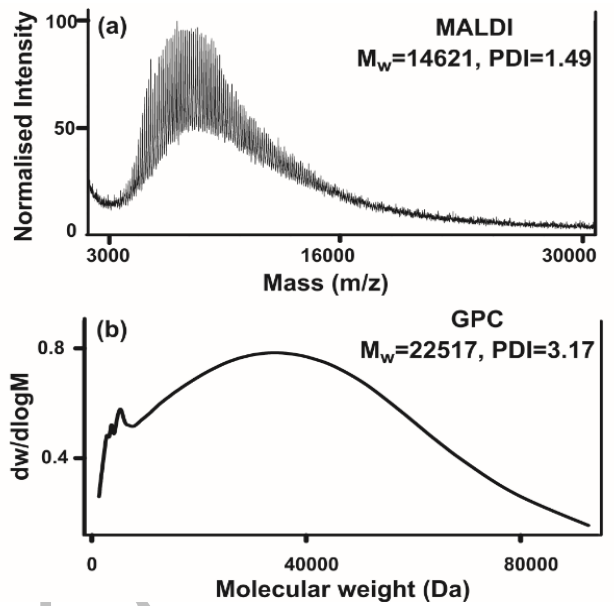

FIGURE 1 (a) MALDI mass spectra of P2 and (b) GPC spectra of P2.

One factor that accounts for this difference is that no adequate standard is similar to $\mathrm{P} 2$ in the direct correction between retention time and molecular mass in GPC, which leads to the overestimation of the MW of $\mathrm{CPEs}^{32,}{ }^{33}$ In MALDI, no polymer standards are essential to calibrate the instrument, which potentially offers accurate mass determination of a polymer. Besides, due to a moderate resolution, structural information about end groups of CPEs cannot be analysed by GPC spectra, while MALDI MS allows not only the calculation of $\overline{M_{n}}, \overline{M_{w}}$ and PDI as in GPC, but also reveals structural information on end groups of CPEs. ${ }^{34}$

\section{End-Group Analysis by M ALDI-TOF MS.}

Seven CPEs were produced through Sonogashira coupling between two monomeric building blocks, diiodo substituted benzene derivative $A$ and diacetylene substituted benzene derivative B. (The structures of factor A and factor $B$ are listed in Table S1.) PRU represents the polymer repeat units of CPEs. E represents end-groups. 


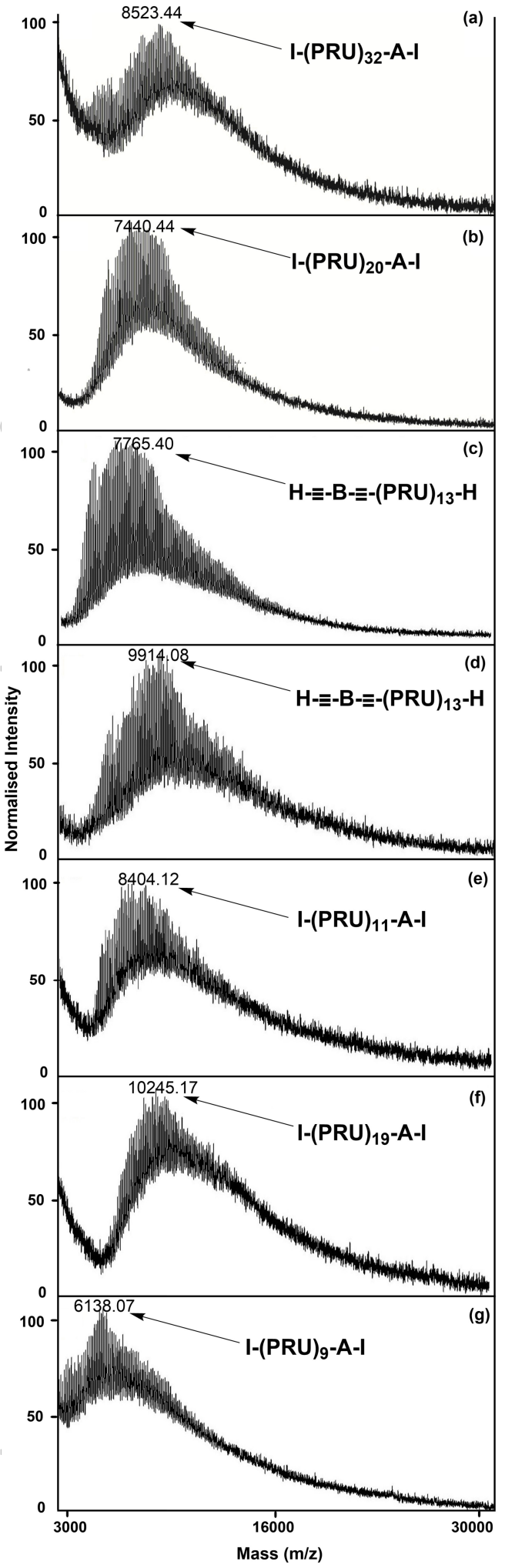

FIGURE 2 M ALDI mass spectra of P1 (a), P2 (b), P3 (c), P4 (d), P5 (e), P6 (f) and P7 (g). in Figure 2(a) corresponds well to $\mathrm{I} / \mathrm{H}$ chain with degree of polymerization of 32 (the calculated mass of this chain is 8088.1). We found that the relative abundances of these types of end-group peaks of seven CPEs were variable. For some CPEs, we could observe end groups terminated by iodine atoms at both chain ends. Information about the end groups of seven CPEs were shown in TABLE S2.

\section{Choice of Matrix}

The success of MALDI experiments on CPEs lies in the choice of matrix, which converts the absorbed laser energy to eject the polymers into the gas phase..$^{15}$ Conjugated polymers have been characterised by MALDI-TOF MS with matrixes, such as $\mathrm{DHB}^{14}, \mathrm{IAA}^{35}$ dithranol, ${ }^{36}$ and $\mathrm{CHCA}^{20,21}$. In this study, six matrixes, including SA, IAA, DHB, THAP, CHCA, and HABA, were examined to characterise seven CPEs. In the case of P2, the MALDI results were shown in Figure S1. Only DHB was found to give good MALDI results, while all other matrixes produced mass results with poor sensitivity and resolution. We believe that CPEs with DHB resulted in well-packed microcrystals as the solvent evaporated, which ensured the laser energy to eject the polymers to gas-phase species. For other matrixes, there is a good possibility that CPEs might precipitate before matrix crystal formation. Hence, DHB was chosen as the matrix for all of seven CPEs samples.

\section{The Effect of P/M/CR Ratio}

$M$ atrix to polymer volume ratios were optimized for different CPEs. The most suitable volume ratios were 5/5/1 (P/M/CR) for anionic polymer (P1-P4) and 5/50/1 (P/M/CR) for cationic polymer (P5-P7), respectively. In addition, the quality of mass spectra was affected by polymer structures. Anionic CPEs (P1-P4) are more easily ionised to produce good results than cationic CPEs (P5-P7) because the $\mathrm{Ag}+$ ions bound to the anionic polymer contributed to ionisation of anionic CPEs. The ratio $1 / 1$ (M/P) gave unsuccessful MALDI results for P5 to P7 while the ratio as high as 10/1 (M/P) was suitable for 
cationic polymers to obtain MALDI results with high resolution. Since P5, P6 and P7 might precipitate before matrix crystal formation due to the electrostatic repulsion between $\mathrm{Ag}^{+}$and positive charges of the three CPEs, a higher matrix ratio was needed to incorporate the CPES into the matrix crystals as the solvent evaporates.

\section{The Choice of CR}

Figure 3 presents the M ALDI mass spectra of $P 2$ with different CR solutions. Figure 3a with LiTFA as $C R$ shows poor signal intensity and high baseline-noise, while Figure $3 c$ obtained with AgTFA as $C R$ shows high signal/noise $(\mathrm{S} / \mathrm{N})$ ratio and resolution. TFA was also utilised as $C R$ and the $\mathrm{S} / \mathrm{N}$ ratios was significantly lower than that with AgTFA. These results suggest that the predominant ionisation mechanism for CPE systems can be attributed to added salt solutions, where photoionization plays a major role in the ionisation process. The peak mass of $[\mathrm{X}+\mathrm{Ag}]^{+},[\mathrm{X}+\mathrm{Na}]^{+}$and $[\mathrm{X}+\mathrm{H}]^{+}$can always be found in the MALDI mass spectra of CPEs. Since

TABLE 3 Characterization Data of Three Batches of P3 the MWD of CPEs is a Gaussian distribution, the complex of CPEs and added salts are widely distributed on the mass spectra.

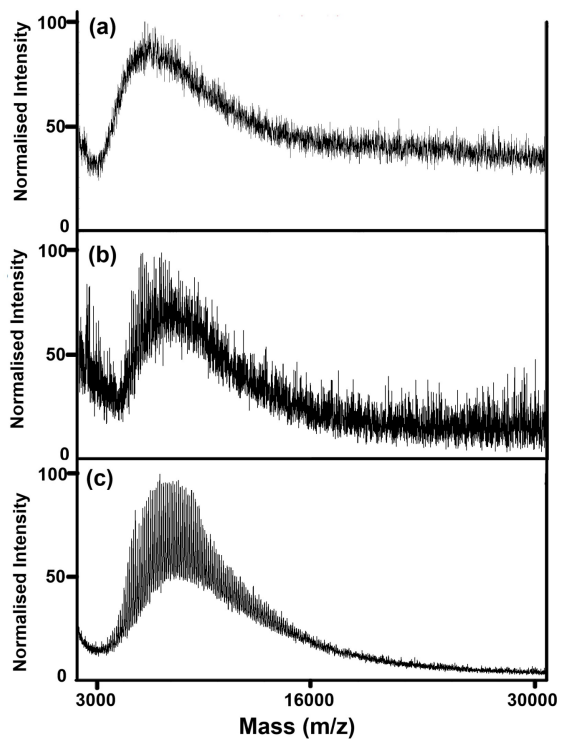

FIGURE 3 MALDI mass spectra of P2 using DHB as the matrix with different CRs. (a) LiTFA, (b)TFA and (c) AgTFA.

\begin{tabular}{|c|c|c|c|c|c|c|c|}
\hline Polymer & $\overline{M_{n}}(D a)$ & $\overline{\mathrm{M}_{\mathrm{w}}}(\mathrm{Da})$ & PDI & $\overline{X_{n}}$ & $\lambda_{\text {max }}^{\text {abs }}(n m)$ & $\lambda_{\max }^{\mathrm{em}}(\mathrm{nm})$ & $\operatorname{Time}^{\mathrm{b}}(\mathrm{h})$ \\
\hline P3-1 & 13912 & 21120 & 1.59 & 24 & 378 & 427 & 18 \\
\hline P3-2 & 14788 & 22182 & 1.50 & 26 & 382 & 428 & 24 \\
\hline P3-3 & 22536 & 45973 & 2.04 & 39 & 409 & 428 & 48 \\
\hline
\end{tabular}

${ }^{a}$ Obtained according to formula PDI $=\overline{M_{w}} / \overline{M_{n}}$

${ }^{\mathrm{b}}$ Polymerization time

\section{Effect of MW on Optical Properties}

The MW of CPEs have important influence on their optical behaviour. In order to study the effect of chain length on the optoelectronic properties of CPEs, three batches of P3 (P3-1, P3-2, and P3-3) were synthesized with various polymerisation time as $18 \mathrm{~h}, 24 \mathrm{~h}$, and $48 \mathrm{~h}$, respectively. Table 3 shows the characterization data of three batches of P3. As the lengthening of polymerisation time, the $\overline{M_{n}}$ of $P 3$ increase from $13.9 \times 10^{3} \mathrm{Da}$ to $22.5 \times 10^{3} \mathrm{Da}$ while the $\overline{M_{w}}$ of P3 increase from $21.1 \times 10^{3}$ Da to $45.9 \times$ $10^{3} \mathrm{Da}$. Figure 4 displays the absorption and emission spectra with chain lengths of 24 (P3-1), 26 (P3-2) and 39 (P3-3) RUs, respectively. Optical absorption was obtained in aqueous solutions in order to monitor the bathochromic shift that occurs due to different degrees of polymerization. The absorption maxima $\left(\lambda_{\max }^{\text {abs }}\right)$ for P3-1, P3-2 and P3-3 are $378 \mathrm{~nm}, 382 \mathrm{~nm}$ and $409 \mathrm{~nm}$, respectively. As the length of the polymer chain increases, the $\pi-\pi^{*}$ peak shifts toward longer wavelengths due to the increasing contribution of segments with longer 


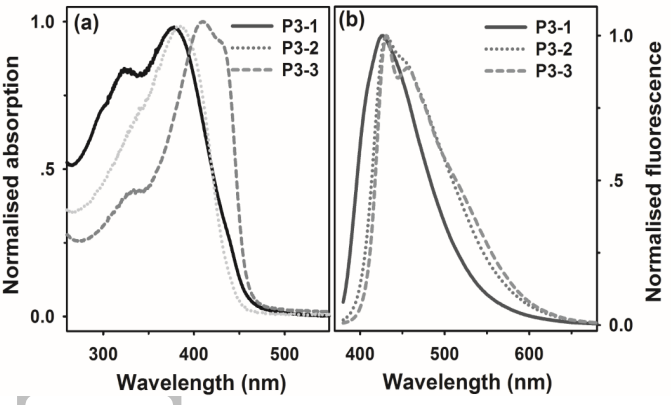

FIGURE 4 (a) Normalised absorption spectra and (b) fluorescence spectra of P3-1 (1 $\mu \mathrm{M})$, P3-2 (1 $\mu \mathrm{M})$, and P3-3 (1 $\mu \mathrm{M})$ in aqueous solutions.

conjugation lengths. ${ }^{37-39}$ Emission spectra of three batches of P3 present no obvious fluorescence peak shifts as the chain length extended. They display strong fluorescence in the blue-green region with excitation at $360 \mathrm{~nm}$. The emission maxima peaks ( $\lambda_{\max }^{\mathrm{em}}$ ) of P3-1 appears at $427 \mathrm{~nm}$. Meanwhile, the $\lambda_{\max }^{\mathrm{em}}$ of P3-2 displays only $1 \mathrm{~nm}$ red-shift to P3-1. P3-3 exhibits emission spectra which are indistinguishable from P3-2 within the resolution of our measurements, even though P3-3 has approximately twice the RU. The insensitivity of P3 to MW was revealed in emission spectra.

Additional information concerning the effect of lateral substituents on the photophysical properties of CPEs comes from the UV-Vis absorption spectra of $\mathrm{P} 2$ and $\mathrm{P} 3$, which are presented in Figure 5. In methanol, the $\lambda_{\max }^{\text {abs }}$ of $\mathrm{P} 2$ and P3 are at $396 \mathrm{~nm}$ and $384 \mathrm{~nm}$, respectively. $P 2$ shows $12 \mathrm{~nm}$ redshift relative to P3. In aqueous solution, the $\lambda_{\max }^{\text {abs }}$ at $426 \mathrm{~nm}$ of $\mathrm{P} 2$ are $44 \mathrm{~nm}$ red-shifted relative to $\mathrm{P3}$, of which
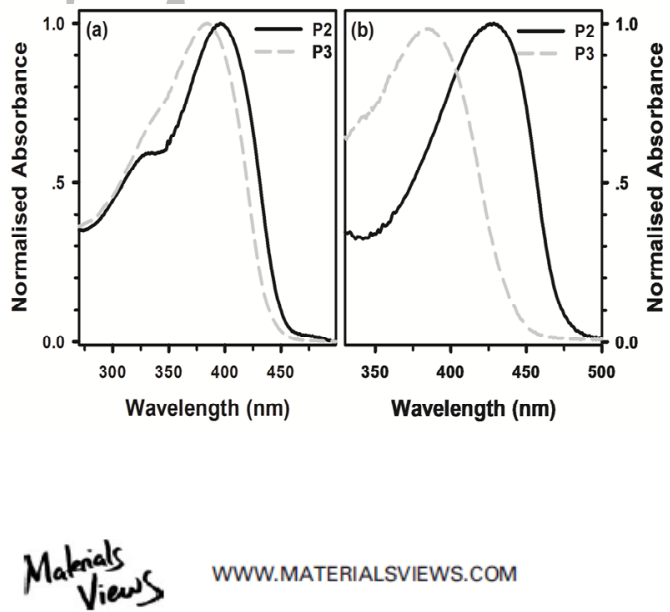

WWW.MATERIALSVIEWS.COM
FIGURE 5 Normalised UV-Vis absorption spectra of P2 and P3 in (a) methanol and (b) aqueous solutions.

the $\lambda_{\max }^{\text {abs }}$ is at $382 \mathrm{~nm}$. As the RUs of P2 is closer to $\mathrm{P} 3$, the difference in the UV-V is absorption is apparently attributed to distinct structure of lateral substituents of P2 and P3. W ith only two negative charges on each RU, P2 shows more aggregation than $\mathrm{P} 3$ in aqueous solution, which lead to the red-shirt of $\lambda_{\max }^{\text {abs }}$ on the UV-Vis absorption.

\section{Effect of MW on Quenching Properties}

The fluorescence quenching response ability of three batches of P3 were evaluated by methyl viologen $\left(\mathrm{MV}^{2+}\right){ }^{40}$ which are shown in Figure 6. The quenching mechanism is photo-induced electron transfer through the complexation of the polymers and $\mathrm{MV}^{2+}{ }^{41}$ In the CPE-MV ${ }^{2+}$ systems, P3-3 demonstrated the most efficient quenching by $\mathrm{MV}^{2+}$ among the three batches of $\mathrm{P3}$, presumably due to efficient energy migration in the backbone. At the same $\mathrm{MV}^{2+}$ concentration $(c=1.0 \mu \mathrm{M})$, about $69 \%$ of the fluorescence of P3-3 were quenched, whereas $18 \%$ and $63 \%$ of fluorescence were quenched for P3-1 and P3-2, respectively. Although the SV plots of P3-1 and P3-2 exhibited a linear correlation at the quencher concentration ( $c=0$ - $2.0 \mu \mathrm{M}$ ), a superlinear behaviour (upward curvature) was shown in the SV plot of P3-3 due to the efficient singlet exciton migration within P3-3. These features were reported for other high MW poly(phenylene ethynylene)s, which was

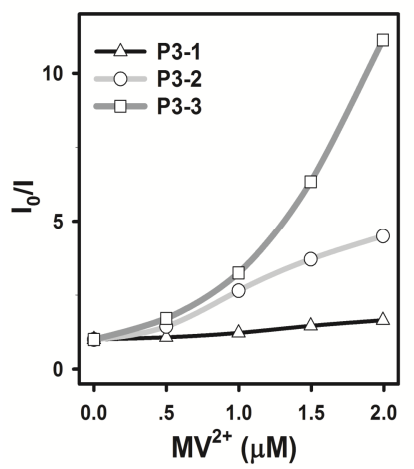

JOURNAL OF POLYMER SCIENCE PART A: POLYMER CHEMISTRY 
FIGURE 6 Stern-Volmer (SV) quenching of P3-1, P3-2, and P3-3 by $\mathrm{MV}^{2+}$ in water. $\left(\mathrm{I}_{0}\right.$ and $\mathrm{I}$ represent the integral area of emission of $\mathrm{P} 3$ without $\mathrm{MV}^{2+}$ and with $\mathrm{MV}^{2+}$, respectively.)

believed to be due to the excitions' elocalisation and rapid interchain diffusion. ${ }^{41-43} 44$

\section{CONCLUSIONS}

MW and MWD of seven CPEs synthesized through Sonogashira coupling reaction were determined using MALDI-TOF MS. Instrumental parameters and measurement conditions were optimized. Combinations of 5/5/1 (P/M/CR) for anionic polymer (P1-P4) and 5/50/1 (P/M/CR) for cationic polymer (P5-P7) were suitable to characterize CPEs with DHB as the matrix and AgTFA as the cationisation reagent. Compared to GPC, M ALDI-TOF-M S provided more accurate structural information about CPEs on repeat units, end-group structure and MW. CPEs with various end groups could be found in the M ALDI mass spectra, including $\mathrm{H} / \mathrm{I}, \mathrm{H} / \mathrm{A}-\mathrm{I}$ and $\mathrm{I} / \mathrm{A}-\mathrm{I}$ etc. Further physical characterization and quenching studies were carried out to explore the effects of MW. CPES showed an obvious red-shift in UV$V$ is absorption spectra due to a lower energy gap between polymers' conduction band and valence band when the conjugation lengths of polymers increased. The $\mathrm{MV}^{2+}$ showed a higher quenching efficiently to CPEs with longer conjugation lengths because of the efficient singlet exciton migration within the polymer.

\section{ACKNOWLEDGEMENTS}

We appreciate Prof. Kirk S. Schanze for providing some of the polymer samples used in this study. This work is supported by grants from the National Natural Science Foundation of China (No. 21572115) and Shenzhen Municipal Government (JCYJ20160301153959476 and JCY20160324163734374).

\section{REFERENCES AND NOTES}

1 C. Zhu, L. Liu, Q. Yang, F. Lv, S. Wang, Chem. Rev. 2012, 112, 4687-4735.
2 S. W. Thomas, G. D. Joly, T. M. Swager, Chem. Rev. 2007, 107, 1339-1386.

3 D. T. McQuade, A. E. Pullen, T. M. Swager, Chem. Rev. 2000, 100, 2537-2574.

$4 \mathrm{H}$. Jiang, P. Taranekar, J. R. Reynolds, K. S. Schanze, Angew. Chem. Int. Ed. 2009, 48, 43004316.

5 X. Zhao, M. R. Pinto, L. M. Hardison, J. Mwaura, J. Müller, H. Jiang, D. Witker, V. D. Kleiman, J. R. Reynolds, K. S. Schanze, Macromol. 2006, 39, 6355-6366.

6 X. Zhao, H. Jiang, K. S. Schanze, Macromol. 2008, 41, 3422-3428.

7 C. Jackson, B. Larsen, C. M cEwen, Anal. Chem. 1996, 68, 1303-1308.

8 A. Bootz, T. Russ, F. Gores, M. Karas, J. Kreuter, Eur. J. Pharm. Biopharm 2005, 60, 391399.

9 N. Sakurada, T. Fukuo, R. Arakawa, K. Ute, K. Hatada, Rapid Commun. Mass. Spectrom. 1998, 12, 1895-1898.

10 R. D. Davis, W. L. Jarrett, L. J. Mathias, Polymer 2001, 42, 2621-2626.

11 M. Tabata, V. Boucard, D. Ades, A. Siove, T. Sone, T. Seino, Y. Mawatari, Macromol. 2001, 34, 8101-8106.

12 T. Gruendling, S. Weidner, J. Falkenhagen, C. Barner-Kowollik, Polym. Chem. 2010, 1, 599617.

13 Y. H. Wen, P. C. Lin, C. C. Hua, S. A. Chen, J. Phys. Chem. B 2011, 115, 14369-14380.

$14 \mathrm{H}$. J. Rader, J. Spickermann, M. Kreyenschmidt, K. Mullen, Macromol. Chem. Phys. 1996, 197, 3285-3296.

15 S. D. Hanton, Chem. Rev. 2001, 101, 527-569. 16 L. Przybilla, V. Francke, H. J. Rader, K. M ullen, Macromol. 2001, 34, 4401-4405.

$17 \mathrm{H}$. Brandt, T. Ehmann, M. Otto, J. Am. Soc. Mass. Spectrom. 2010, 21, 1870-1875.

18 M. Doycheva, E. Berger-Nicoletti, F. Wurm, H. Frey, Macromol. Chem. Phys. 2010, 211, 3544.

19 S. Weidner, P. Knappe, U. Panne, Anal. Bioanal. Chem. 2011, 401, 127-134.

20 K. P. R. Nilsson, J. D. M. Olsson, P. Konradsson, O. Inganäs, Macromol. 2004, 37, 6316-6321. 
21 K. P. R. Nilsson, J. D. M. Olsson, F. Stabo-Eeg, $M$. Lindgren, $P$. Konradsson, 0 . Inganas, M acromol. 2005, 38, 6813-6821.

22 X.-T. Hao, T. Ryan, M. F. Bailey, T. A. Smith, Macromol. 2009, 42, 2737-2740.

23 Y. Wu, Y. Tan, J. Wu, S. Chen, Y. Z Chen, X. Zhou, Y. Jiang, C. Tan, ACS Appl. Mater. Interfaces 2015, 7, 6882-6888.

24 Q. L. Fan, Y. Zhou, X. M. Lu, X. Y. Hou, W. Huang, M acromol. 2005, 38, 2927-2936.

25 Y. Tang, E. H. Hill, Z. Zhou, D. G. Evans, K. S. Schanze, D. G. Whitten, Langmuir. 2011, 27, 4945-4955.

26 K. Tanaka, H. Waki, Y. Ido, S. Akita, Y. Yoshida, T. Yoshida, T. Matsuo, Rapid Commun. M ass. Spectrom. 1988, 2, 151-153.

27 M. Remmers, B. Muller, K. Martin, H. J. Rader, W. Kohler, Macromol. 1999, 32, 10731079.

28 S. Hanton, Chem. Rev. 2001, 101, 527-570.

29 T. D. McCarley, C. O. Noble, C. J. DuBois, R. L. M cCarley, M acromol. 2001, 34, 7999-8004.

30 K. P. R. Nilsson, J. D. M. Olsson, P. Konradsson, O. Inganas, Macromol. 2004, 37, 6316-6321.

31 T. Yokozawa, T. Asai, R. Sugi, S. Ishigooka, S. Hiraoka, J. Am. Chem. Soc. 2000, 122, 83138314.

32 G. Montaudo, D. Garozzo, M. S. Montaudo, C. Puglisi, F. Samperi, Macromol. 1995, 28, 7983-7989.

33 S. Holdcroft, J. Polym. Sci., Part B: Polym. Phys. 1991, 29, 1585-1588.

34 H. Pasch, F. Gores, Polymer 1995, 36, 19992005.

35 H. Chen, M. Y. He, J. Pei, B. Liu, Anal. Chem. 2002, 74, 6252-6258.

36 J. S. Liu, R. S. Loewe, R. D. McCullough, M acromol. 1999, 32, 5777-5785.

37 P. V. James, P. K. Sudeep, C. H. Suresh, K. G. Thomas, J. Phys. Chem. A 2006, 110, 4329-4337. 38 L. T. Liu, D. Yaron, M. I. Sluch, M. A. Berg, J. Phys. Chem. B 2006, 110, 18844-18852.

39 G. Castruita, E. Arias, I. Moggio, F. Perez, D. Medellin, R. Torres, R. Ziolo, A. Olivas, E. Giorgetti, M. Muniz-Miranda, J. Mol. Struct. 2009, 936, 177-186.
40 K. Haskins-Glusac, M. R. Pinto, C. Tan, K. S. Schanze, J. Am. Chem. Soc. 2004, 126, 1496414971.

41 L. H. Chen, D. W. McBranch, H. L. Wang, R. Helgeson, F. Wudl, D. G. Whitten, Proc. Natl. Acad. Sci. U. S. A. 1999, 96, 12287-12292.

42 B. S. Harrison, M. B. Ramey, J. R. Reynolds, K. S. Schanze, J. Am. Chem. Soc. 2000, 122, 85618562.

43 C. Tan, M. R. Pinto, K. S. Schanze, Chem. Commun. 2002, 446-447.

44 C. Tan, E. Atas, J. G. M üller, M. R. Pinto, V. D. Kleiman, K. S. Schanze, J. Am. Chem. Soc. 2004, $126,13685-13694$. 


\section{University Library}

\section{- M M I N E R VA A gateway to Melbourne's research publications}

Minerva Access is the Institutional Repository of The University of Melbourne

Author/s:

Wu, J;Tan, C;Zhou, X;Tan, Y;Yang, P;Jiang, Y

Title:

Molecular weight analysis of water-soluble poly(phenylene ethynylene)s using MALDI-TOF MS

Date:

2017-08-01

Citation:

Wu, J., Tan, C., Zhou, X., Tan, Y., Yang, P. \& Jiang, Y. (2017). Molecular weight analysis of water-soluble poly(phenylene ethynylene)s using MALDI-TOF MS. JOURNAL OF POLYMER SCIENCE PART A-POLYMER CHEMISTRY, 55 (15), pp.2537-2543. https://doi.org/10.1002/ pola.28650.

Persistent Link:

http://hdl.handle.net/11343/292922 